\title{
Orthogonality catastrophe in a composite fermion liquid
}

\author{
Darren J. T. Leonard, T. Portengen, V. Nikos Nicopoulos and Neil F. Johnson. \\ Department of Physics, \\ Clarendon Laboratory, \\ Oxford University, \\ Oxford OX1 3PU, \\ England.
}

September 11, 2018

\begin{abstract}
We discuss the emergence of an orthogonality catastrophe in the response of a composite fermion liquid as the filling factor $\nu$ approaches $1 / 2 m$, where $m=1,2,3 \ldots$ A tunneling experiment is proposed in which dramatic changes in the I-V characteristic should be observable as $\nu$ is varied. Explicit I-V characteristics calculated within the so-called Modified Random Phase Approximation, are provided for $\nu=1 / 3 \rightarrow 1 / 2$.
\end{abstract}

\section{Article}

Composite fermion theory [1, 2, 34 has been remarkably successful in explaining the fractional quantum Hall effect in terms of the integer quantum Hall effect of a composite fermion metal [4, 5]. Within this theory, the ground state of a two-dimensional electron gas (2DEG) at even-denominator filling factors $\nu=1 / 2 m, m=1,2,3 \ldots$, is a compressible Fermi liquid containing composite fermions which experience zero effective magnetic field $B^{\star}$ [1, 2, 3]. Various recent experiments on systems near $\nu=1 / 2$ have indeed been interpreted in terms of a Fermi sea of composite fermions [4, 5]. Further confirmation of the details of the theory as $\nu \rightarrow 1 / 2 m$ is still however desirable. In this preprint, we propose an experiment to probe the emergence of the Fermi surface in a com- posite fermion liquid as $\nu \rightarrow 1 / 2 m$. Mono-energetic electrons are allowed to tunnel into a quantum dot placed close to the 2DEG. We find the signature of the presence of a Fermi surface at $\nu=1 / 2 m$ to be a dramatic 'orthogonality catastrophe' in the tunneling current (I) as a function of the gate voltage (V) of the dot. For $\nu \neq 1 / 2 m$, strong oscillatory signatures in the I-V characteristic are predicted - these signatures are $\nu$-dependent and can be used to deduce the composite fermion effective mass. In addition, the spectrum for odd-denominator fractions will yield valuable information about the 'gapfull' excitations of these states.

Our proposed experiment is analogous to inverse photoemission spectroscopy (IPS) [6, 7] experiments on ordinary metals, with the atomic core level replaced by a quantum dot. In an IPS experiment, a free electron falls into a hole in an atomic core state thereby emitting a photon of energy $\omega_{0}$. The intensity of the emitted photon is identically zero at the threshold energy $\omega_{0}$. This is a consequence of the so-called 'orthogonality catastrophe' (OC); the transition involved is forbidden because the initial and final states are orthogonal [6, 7, 8, 9, 10.

Figure 11 provides a schematic illustration of our proposed experiment. Quantum dot A acts as an electron monochromator, because only source electrons with energy $\epsilon$ can resonantly tunnel to A. An electron in A will resonantly tunnel to quantum dot B only if there are states available with energy $\epsilon$. In 
the absence of the 2DEG the density of states of B is a $\delta$-function at energy $\epsilon_{0}$, and tunneling occurs only when $\epsilon=\epsilon_{0}$. However, the presence of the 2DEG means that the density of states is asymmetrically broadened to higher energies due to the neutral excitations in the 2DEG induced by the filled dot - this implies that tunneling can occur if $\epsilon>\epsilon_{0}$. The electron can then tunnel out into the drain lead to be measured as a current, determined by the tunneling rates $\gamma_{a}, \Gamma$, and $\gamma_{b}$. The current $I$ is measured as function of the gate voltage $V$ controlling the difference between the energy $\epsilon$ of the injected electron and the energy $\epsilon_{0}$ of dot B. This resonant tunneling is similar to IPS with a zero energy photon, and the analog of the IPS spectrum is the tunneling I-V characteristic; the threshold $V=V_{0}$ in this case is such that $\epsilon_{0}=\epsilon$. Generally, $\epsilon-\epsilon_{0}=e\left(V-V_{0}\right)$, hence the creation of excitations implies that the spectrum is non-zero for $V>V_{0}$.

Once an electron has tunneled into dot B it must reside there for a time greater than the response time of the 2DEG. This implies that the electron tunnels out with rate $\gamma_{b}$ less than the desired resolution, which is typically the composite fermion Landau-level spacing for a filling factor close to $\nu=1 / 2$. The other two rates $\Gamma$ and $\gamma_{a}$ are determined by the following simple argument. The average current can be written as

$$
I=\frac{e}{T}
$$

where $T$ is the total time taken to tunnel from source to drain. In terms of the tunneling rates we have for sequential tunneling

$$
T \sim \frac{1}{\gamma_{a}}+\frac{1}{\Gamma}+\frac{1}{\gamma_{b}} .
$$

In order for the tunneling current to reflect only the density of states of $\operatorname{dot} \mathrm{B}$, we choose the conditions

$$
\gamma_{a} \sim \gamma_{b} \equiv \gamma \quad \Gamma \ll \gamma \quad \gamma \leq \omega_{c}^{\star},
$$

in which case the current is given by

$$
I=e \Gamma=e \Gamma_{0} \gamma \operatorname{Re} \int_{0}^{\infty} d t e^{i e\left(V-V_{0}\right) t / \hbar-F(t)-\gamma t} .
$$

The time-integral in Eq. (4) gives the convolution of the density of states of dot $\mathrm{B}$ with a Lorentzian of width $\gamma$ representing the broadening due to tunneling to the drain. In the absence of the 2DEG, the function $F(t)=0$, and $I\left(V_{0}\right)=e \Gamma_{0}$. The bare tunneling rate $\Gamma_{0}$ is determined solely by the width and height of the barrier between dots $\mathrm{A}$ and $\mathrm{B}$. A suitable value consistent with Eq. (3) is $\hbar \Gamma_{0} \approx 6 \mu \mathrm{eV}$. The density of states of the electron, once it has tunneled to $\operatorname{dot} B$, depends on the excitation spectrum of the 2DEG through the function

$$
F(t)=\int_{0}^{\infty} d \omega \frac{\left(1-e^{-i \omega t}\right)}{\omega^{2}} \rho(\omega)
$$

where

$$
\rho(\omega)=\frac{1}{\hbar} \sum_{\mathbf{q}}|V(\mathbf{q})|^{2} S(\mathbf{q}, \omega)
$$

is the density of single pair-excitations of the $2 \mathrm{DEG}$ due to the sudden appearance of an electron in B. $V(\mathbf{q})$ is the potential experienced by the $2 \mathrm{DEG}$ due to the electron in B [6]. The dynamic structure factor $S(\mathbf{q}, \omega)$ 11] contains information about the excitation spectrum of the 2DEG, and is calculated using the Chern-Simons theory of composite fermions within the modified random phase approximation (MRPA) [3]. We note that in approximating $F(t)$ as above, we are treating the excitations of momentum $\mathbf{q}$ and energy $\omega$ as independent bosons, which is standard in the theory of IPS in ordinary metals. The theory includes all such bosonic excitations exactly to all orders in perturbation theory [6].

The MRPA resolves the conflict of requiring both renormalisation of the composite fermion mass and satisfaction of Kohn's theorem [1, 2, 3, 11]. In the limit where the electron cyclotron energy is large compared with the Coulomb energy, the composite fermion mass is expected to scale as the squareroot of the magnetic field. Using the RPA equations with this renormalised mass leads to satisfying neither Kohn's theorem nor the f-sum rule; the MRPA repairs this within Fermi-liquid theory by adding a Landau interaction term. In our calculations we use a renormalised composite fermion effective mass which scales as the square-root of the magnetic field such 
that

$$
m_{\mathrm{CF}}^{\star}=\frac{4 \pi \epsilon_{0} \epsilon_{r} \hbar^{2}}{0.3 e^{2} l_{\mathrm{c}}},
$$

where the magnetic length is $l_{\mathrm{c}}$ and the dielectric constant $\epsilon_{r}=13$ [1, 2, 3].

There are three essential features to be incorporated in the experimental design (see Fig. 1). First, the probe dot B must be separated from the plane of the 2DEG by a barrier which is sufficiently high to prevent tunneling between B and the 2DEG. However, B must be as close to the 2DEG as possible because the Fourier transform of the potential experienced by the 2DEG due to an electron at B decays exponentially with the separation $d$. Second, the source and drain leads and dot A must be far enough away from the 2DEG so that the only potential experienced by the 2DEG comes from the electron at B. Third, the levels in the dots must be well spaced so that only one level contributes to the tunneling; two-electron tunneling will be suppressed because of Coulomb blockade. Our calculations are therefore based on a 2DEG with $10^{15}$ electrons $\mathrm{m}^{-2}$ placed $d=50 \AA$ away from a dot with a confinement length of $50 \AA$.

Figure 2 shows the I-V characteristic calculated for the compressible state with filling factor $\nu=$ $1 / 2$. The solid line does not include any instrumental broadening thereby emphasising the suppression at threshold. The dashed curve shows the current with a Lorentzian broadening of width $\gamma=0.1$ meV; here there is a small current at and below the threshold. The peaked shape is completely different from the power-law which arises for an ordinary metal [6, 7, 8, 9, 10]. The OC is clear, because the threshold current is negligible - the low energy excited states have a very small overlap with the initial state and are strongly suppressed, in complete contrast to those in an ordinary metal. The suppression is a consequence of the diffusive mode which dominates the dynamic structure factor at $\nu=1 / 2$ at low energies and momenta [1]. This mode arises from the scattering of the composite fermions by their attached flux tubes. The sudden appearance of the perturbing potential causes density fluctuations in the 2DEG, which induce modulations in both the scalar and vector potentials resulting from the diago- nal and off-diagonal terms in the Chern-Simons interaction; these potentials further scatter the composite fermions thereby creating more density fluctuations. The result is that these density fluctuations are diffusive, i.e. they decay exponentially with time. The excitations do not exist on a time scale long enough to screen the potential. This situation is very different in an ordinary metal, where the only scattering mechanism available is the Coulomb potential. In this case, the low energy excitations have infinite lifetime, hence they successfully screen the potential in the long-time limit.

The power-law divergence in ordinary metals arises from a density of pair excitations $\rho(\omega)$ which goes linearly with $\omega$ for energies small compared with the Fermi energy [6]. This is not the case for the composite fermion metal, where we find $\rho(\omega)$ varies roughly as $\sqrt{\omega}$. One can derive this result analytically using the single-mode approximation employed by Platzman, He and Halperin [12 in their study of tunneling between two 2DEGs as a function of the bias voltage. It should be noted that our work differs in two ways from that of Ref. [12]. First, and most importantly, their problem involves the charged excitations of the 2DEG because their tunneling process removes an electron from one 2DEG and places it in the other. They treat the added (removed) electron as an infinitely massive foreign particle inserted into the N-electron system, thereby reducing the problem to that of the x-ray edge problem. Our experiment is, by design, analogous to IPS without further need for approximations because the tunneling electron is both distinguishable from the 2DEG and localised. Our experiment therefore strictly probes the neutral excitations of the 2DEG. Second, our work differs in the level of approximation employed for the dynamic structure factor and the subsequent determination of the density of pair excitations. We use the full MRPA dynamic structure factor, which allows us to calculate the I-V characteristic at both odd- and evendenominator filling factors. By contrast, the singlemode approximation used in Ref. 12] is valid only at even-denominator filling factors. Applying the singlemode approximation to our experiment (with $d=0$ ) 
we predict a current of the form

$$
I \propto \frac{1}{\sqrt{\left(V-V_{0}\right)^{3}}} \exp \left(\frac{\alpha}{V_{0}-V}\right) \quad V>V_{0}
$$

which is in qualitative agreement with the current calculated numerically.

Figures 3, 5 and 5 show the I-V characteristic for the $2 \mathrm{DEG}$ at three odd-denominator filling factors originating from the $\nu=1 / 2$ state. A state at $\nu=p /(2 m p+1)$ can be described in terms of composite fermions at effective filling factor $\nu^{\star}=p$ interacting with flux tubes carrying $2 m$ magnetic flux quanta. We have considered the cases where $m=1$ and $p=1,3$, and 7 , which correspond to the fractions $\nu=1 / 3,3 / 7$ and $7 / 15$ respectively. The I-V characteristic at $\nu=p /(2 m p+1)$ differs in two important respects from that at $\nu=1 / 2$. First, there is now no orthogonality catastrophe, because there is a gap to excitations. While in the absence of the 2DEG the threshold current is $e \Gamma_{0}$, the presence of the $2 \mathrm{DEG}$ reduces the threshold current by a factor of $\exp (-L)$, where

$$
L=\int_{0}^{\infty} d \omega \frac{\rho(\omega)}{\omega^{2}}
$$

is the Debye-Waller factor. This current decreases as the effective magnetic field is reduced because $L$ increases, signifying the reduction in the overlap of the initial state and final ground state; in the limit of zero effective field $L \rightarrow \infty$, implying that the overlap tends to zero yielding the OC. Second, the I-V spectrum develops oscillations superimposed on an envelope similar in shape to the I-V curve for $\nu=1 / 2$. The period of these oscillations is given by the composite fermion cyclotron frequency $\omega_{\mathrm{c}}^{\star}=e B^{\star} / m_{\mathrm{CF}}^{\star}$, where $B^{\star}=n_{\mathrm{e}} h / e p$. A measurement of this period can thus provide an experimental test of the validity of Eq. (7).

At high effective fields $B^{\star}$, such as $\nu^{\star}=1(\nu=$ $1 / 3)$, the oscillations dominate the spectrum and the threshold current is large because $L$ is small. As $B^{\star}$ is reduced $L$ increases, thus the threshold current decreases and the envelope function becomes more distinct. As expected the $B^{\star} \rightarrow 0$ limit resembles the $B^{\star}=0$ calculation giving further confidence in our results. The transition from high to low effective field displayed in Figs. 2-5 is quite dramatic - the peak at threshold changes to a complete suppression. We now mention the shape of $\rho(\omega)$ away from $\nu=1 / 2 m . \rho(\omega)$ reflects the gapfull nature of the excitations, containing peaks separated by $\omega_{\mathrm{c}}^{\star}$. When $B^{\star}$ is small, $\rho(\omega)$ has an envelope which goes roughly as $\sqrt{\omega}$ for low energies and hence tends towards the $B^{\star}=0$ (i.e. $\nu=1 / 2 m$ ) shape.

We now compare the response of an ordinary metal in a weak magnetic field with the composite fermion system described above [6, 9]. The I-V spectrum for ordinary electrons in zero magnetic field diverges as a power-law, being identically zero at threshold. On applying a magnetic field the spectrum develops oscillations with a period equal to the cyclotron frequency but retains the divergent shape as an envelope. Since there is now a gap to excitations, there is no $\mathrm{OC}$ and $I\left(V_{0}\right)=e \Gamma_{0} \exp (-L)$. However, even in the high field limit the power-law shape is retained in the envelope of the oscillations. Hence the only changes in the overall shape of the I-V curve as the field is increased are the appearance of oscillations and the disappearance of the orthogonality catastrophe. This is in sharp contrast to the present case of composite fermions where, as shown above, the near-threshold behaviour changes from being completely suppressed in zero effective field (e.g. $\nu=1 / 2$ ), to being highly peaked, at large effective fields (e.g. $\nu^{\star}=1$, and hence $\nu=1 / 3$ ).

We thank B. I. Halperin and S. H. Simon for useful discussions. We acknowledge the financial support of EPSRC through a Studentship (D.J.T.L) and EPSRC grant No. GR/K 15619.

\section{References}

[1] B. I. Halperin, P. A. Lee and N. Read, Phys. Rev. B 47, 7312 (1993).

[2] A. Stern and B. I. Halperin, Phys. Rev. B 52, 5890 (1995); Surf. Sci. 362, 42 (1996).

[3] S. H. Simon and B. I. Halperin, Phys. Rev. B 48, 17368 (1993); 50, 1807 (1994); S. He, S. H. Simon and B. I. Halperin, ibid. 50, 1823 (1994). 
[4] R. R. Du, H. L. Stormer, D. C. Tsui, L. N. Pfeiffer and K. W. West, Phys. Rev. Lett. 70, 2944 (1993); D. R. Leadley, R. J. Nicholas, C. T. Foxon, and J. J. Harris, ibid. 72, 1906 (1994); Perspectives in Quantum Hall Effects, edited by S. Das Sarma and A. Pinczuk (WileyInterscience, New York 1997), Chap. 10.

[5] R. L. Willett, M. A. Paalanen, R. R. Ruel, K. W. West, L. N. Pfeiffer and D. J. Bishop, Phys. Rev. Lett. 54, 112 (1990); R. L. Willett, R. R. Ruel, K. W. West and L. N. Pfeiffer, ibid. 71, 3846 (1993).

[6] G. D. Mahan, Many Particle Physics, 2nd ed. (Plenum, New York, 1990), Chap. 8.

[7] Unoccupied electronic states : fundamentals for $X A N E S, E E L S, I P S$ and BIS, edited by J.C. Fuggle and J.E. Inglesfield (Springer-Verlag, Berlin), Chap. 1.

[8] K. A. Matveev and A. I. Larkin, Phys. Rev B 46, 15337 (1992).

[9] T. Uenoyama and L. J. Sham, Phys. Rev. Lett. 65, 1048 (1990).

[10] P. Nozières and C. T. De Dominicis, Phys. Rev. 178, 1097 (1969).

[11] D. Pines and P. Nozières, The Theory of Quantum Liquids: Volume I, (W. A. Benjamin, Inc., New York 1966).

[12] S. He, P. M. Platzman and B. I. Halperin, Phys. Rev. Lett. 71, 777 (1993).
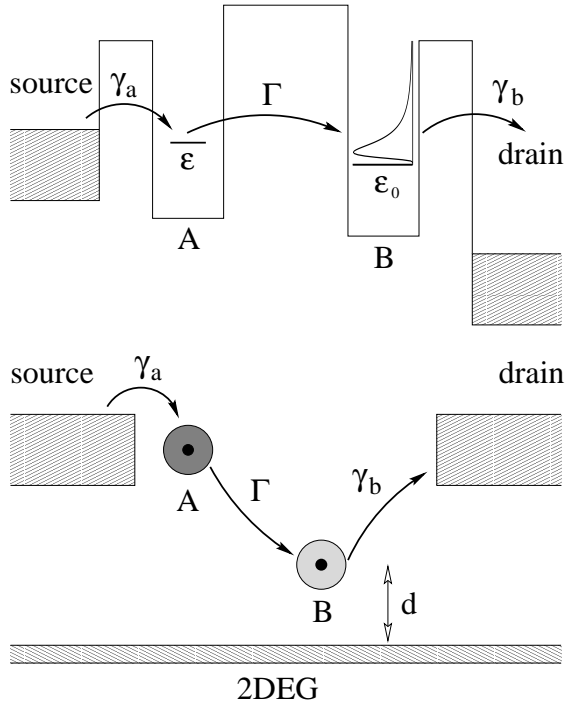

Figure 1: Schematic diagram of the junction. A gate voltage $V$ is applied to dot B to alter the energy of dot $\mathrm{B}$ with respect to $\mathrm{A}$. The source-drain voltage is kept fixed. The plane of the 2DEG is perpendicular to the page.

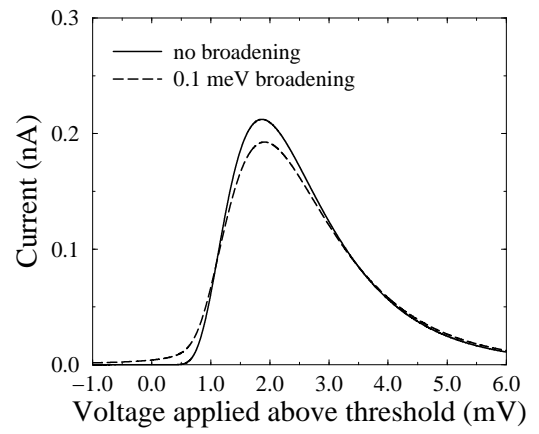

Figure 2: Current at $\nu=1 / 2$ as a function of the voltage applied above threshold, i.e. $V-V_{0}$. Solid curve: spectrum in limit of perfect resolution, i.e. no instrumental broadening. Dashed curve: current with a broadening of $0.1 \mathrm{meV}$. 


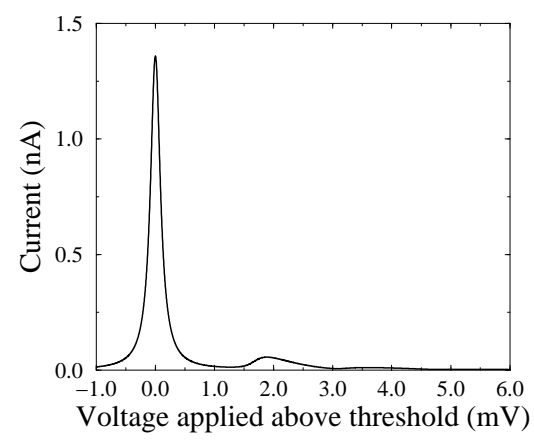

Figure 3: Current calculated for $\nu=1 / 3$ as a function of the voltage applied above threshold, i.e. $V-V_{0}$. Finite broadening as in Fig. 2.

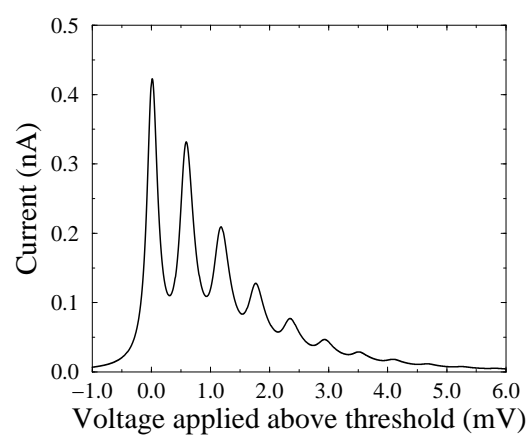

Figure 4: Current at $\nu=3 / 7$ as a function of $V-V_{0}$. Finite broadening as in Fig. 2.

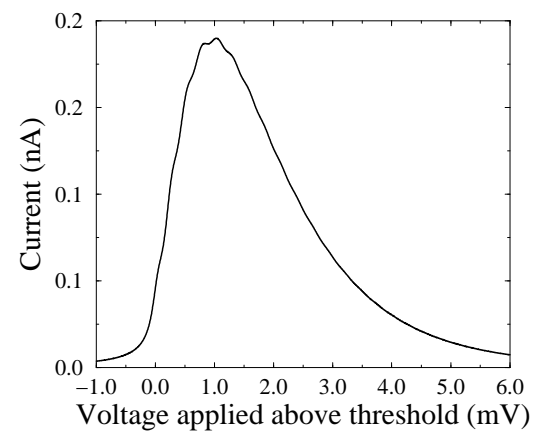

Figure 5: Current at $\nu=7 / 15$ as a function of $V-V_{0}$. Finite broadening as in Fig. 2. 